\title{
Abortamentos na macrorregião leste do Maranhão: fatores socioeconômicos e clínicos
}

Abortions in the eastern macro-region of Maranhão: socioeconomic and clinical factors

Abortos en el macrorregión oriental de Maranhão: factores socioeconómicos y clínicos

\section{Resumo}

O presente artigo visa analisar a situação de abortamentos ocorridos na macrorregião leste do Maranhão e caracterizar o perfil socioeconômico e demográfico dos dados no período de 2010 a 2018. Realizou-se um estudo descritivo de série temporal de base populacional que descreveu o cenário do aborto utilizando dados públicos de sistemas de informação do Brasil - DATASUS (informação do Sistema Único de Saúde (SUS)) e SIH (Sistema de Internação Hospitalar). As evidências da presente pesquisa sugerem que o abortamento na macrorregião em estudo é mais preponderante no município de Timon correspondendo a 19,91\% dos óbitos fetais, em mulheres jovens de 15 a 19 anos e de baixa ou média escolaridade tendo gravidez do tipo única e tipo de parto vaginal com $83,11 \%$. Há também prevalência de óbitos relacionados à fatores maternos equivalendo a 39,82\% dos óbitos e consoante a categoria da Classificação Internacional de Doenças CID-10 por hipóxia intrauterina com $29,87 \%$. Observa-se também a presença de muitos casos ignorados refletindo a importância do completo preenchimento dos registros hospitalares, desta forma, esta pesquisa dedica-se a auxiliar na proteção, prevenção e promoção da saúde da mulher e do recém-nascido. Palavras-chave: Aborto; Fatores socioeconômicos; Saúde da mulher.

\footnotetext{
Abstract

This article aims to analyze the situation of abortions in the eastern macro-region of Maranhão and characterize the socioeconomic and demographic profile of the data in the period from 2010 to 2018. A descriptive population-based time series study was carried out that described the scenario of abortion using public data from information systems in Brazil - DATASUS (information from the Unified Health System (SUS)) and SIH (Hospital Hospitalization System). The evidence from the present research suggests that abortion in the macro-region under study is more prevalent in the municipality of Timon, corresponding to $19.91 \%$ of fetal deaths, in young women aged 15 to 19 years and with low or medium education, having a single-type pregnancy and type of vaginal delivery with $83.11 \%$. There is also a prevalence of deaths related to maternal factors, equivalent to $39.82 \%$ of deaths and according to the category of the
} 
International Classification of Diseases ICD-10 due to intrauterine hypoxia with $29.87 \%$. It is also observed the presence of many ignored cases, reflecting the importance of completely filling out hospital records, thus, this research is dedicated to assisting in the protection, prevention and promotion of the health of women and of the newborn.

Keywords: Abortion; Socioeconomic factors; Women's health.

\section{Resumen}

Este artículo tiene como objetivo analizar la situación de los abortos en la macrorregión oriental de Maranhão y caracterizar el perfil socioeconómico y demográfico de los datos en el período de 2010 a 2018 . Se realizó un estudio descriptivo de serie temporal poblacional que describió el escenario del aborto utilizando datos públicos de los sistemas de información en Brasil - DATASUS (información del Sistema Único de Salud (SUS)) y SIH (Sistema de Hospitalización Hospitalaria). La evidencia de la presente investigación sugiere que el aborto en la macrorregión en estudio es más prevalente en el municipio de Timón, correspondiente al 19,91\% de las muertes fetales, en mujeres jóvenes de 15 a 19 años y con educación baja o media, con embarazo de tipo único y tipo de parto vaginal con $83,11 \%$. También existe una prevalencia de defunciones relacionadas con factores maternos, equivalente al $39,82 \%$ de las defunciones y según la categoría de la Clasificación Internacional de Enfermedades CIE-10 por hipoxia intrauterina con $29,87 \%$. También se observa la presencia de muchos casos ignorados, reflejando la importancia de completar completamente los registros hospitalarios, por lo que esta investigación se dedica a ayudar a proteger, prevenir y promover la salud de la mujer y del recién-nacido.

Palabras clave: Aborto; Factores socioeconómicos; Salud de la mujer.

\section{Considerações Iniciais}

A prática do abortamento vem sendo realizada desde a antiguidade, sendo tratado de formas diferentes em períodos históricos e contextos políticos distintos (Bonassa, et al.,2015). A organização Mundial da Saúde (OMS) define o abortamento como a expulsão ou extração do feto até a $22^{\mathrm{a}}$ semana completa de gestação e/ou peso fetal inferior a $500 \mathrm{~g}$, podendo ser precoce, quando ocorre até a $12^{\mathrm{a}}$ semana de gravidez, ou tardio quando acontece entre a $13^{\mathrm{a}}$ e $22^{\mathrm{a}}$ semanas (Desterro, et al., 2015).

O primeiro registro do aborto foi creditada ao Imperador Chinês Shennong, que, em texto médico datado entre 2.737 e 2.696 a. C, apresenta a receita de um abortífero oral à base de mercúrio. Posteriormente, na Grécia Antiga, Aristóteles, Platão e Hipócrates pensavam diferentemente sobre o tema, os dois primeiros o defendiam como método de controle de natalidade e por razões eugênicas, enquanto Hipócrates colocava-se contra a realização do procedimento. Passando para a história da idade contemporânea, no período pós Primeira Guerra Mundial, em diversos países da Europa Ocidental, houve a opção por políticas severas contra o aborto, devido à escolha por uma política natalista (Henriques, 2016).

Segundo o pensamento de Tomás de Aquino e Santo Augustino, no século IV, o embrião só possuía vitalidade por volta de 40 dias após a concepção, que era quando Deus enviava uma alma ao corpo materno, e isso só acontecia a medida que este corpo estivesse preparado para recebe-la, antes dessa idade, o aborto não era considerado crime. Esse pensamento durou até o ano de 1588, quando leis e doutrinas medievais passaram a considerar os movimentos no ventre da mãe, como parâmetros para justificar quando o aborto deixaria de ser aceitável (Pereira, 2018).

Os anos noventa foram marcados por uma cobertura midiática sintonizada com as controvérsias acerca da legislação brasileira. A partir de 1970, houve uma intensificação, no contexto do movimento feminista, na luta para a reforma do Código Penal no que se referia ao aborto (Silva \& Martins, 2015). Apesar da legalização do aborto sempre ter sido uma das prioridades dos direitos humanos das mulheres, por muitas vezes, o foco teve que ser desviado, pois havia necessidade de lutar por assuntos referentes a métodos contraceptivos e natalidade, já que naquele momento, era mais necessário que o aborto de forma isolada (Fleury, 2016).

Abortos podem ser classificados em seguros, menos seguros ou inseguros, dependendo do método utilizado para sua indução e do profissional responsável pela assistência. Estima-se que, no mundo, sejam realizados anualmente 35 abortos para cada 1.000 mulheres entre 15 e 44 anos. A América Latina se destaca como uma das regiões de maior frequência de 
aborto inseguro (44/1.000), apesar de legislações restritivas na maioria dos seus países, excetuando-se o Uruguai, a Colômbia e Cuba (Azevedo, 2017).

A gravidez precoce e não planejada tem grande influencia nas taxas de morbidade e mortalidade, além do que, o índice de abandono escolar aumenta significativamente com o aumento dos casos de gravidez na adolescência, o que irá contribuir, para o predomínio de baixa ou média escolaridade das mulheres que passam pelo processo de aborto. A juventude em grande parte não possui conhecimento a cerca dos métodos anticonceptivos ou contraceptivos, o que influencia diretamente para o acontecimento de gravidez não planejada e consequentemente o risco de abortos inseguros (Munakampe, et al., 2021).

No Brasil, o aborto é permitido para mulheres com risco de vida, nas situações de gravidez por estupro e, recentemente, na presença de anencefalia fetal. As duas primeiras estão fundamentadas no Artigo 128 do Código Penal e a terceira resulta do julgamento da Arguição de Descumprimento de Preceito Fundamental (ADPF-54), pelo Supremo Tribunal Federal (STF), em 2012, ratificada em decisão do Conselho Federal de Medicina (CFM), também em 2012, que autoriza médicos a interromperem a gravidez de fetos anencefálicos, a pedido da gestante, em qualquer momento da gravidez. Além dessas situações, alvarás judiciais para anencefalia (antes de 2012) e para outras malformações têm sido liberados no Brasil, ampliando as perspectivas do aborto legal (CFM, 2012).

No campo legislativo, muitas já foram as tentativas de modificação do código penal, no que diz respeito à caracterização do aborto enquanto crime, entre elas: a descriminalização do aborto no primeiro trimestre de gestação, a flexibilização dos casos previstos em lei, a realização de abortos legais pelo Sistema Único de Saúde (SUS), entre outros. No período entre 2000 e 2007, 25 projetos de lei foram apresentados com o foco no tema do aborto, desde projetos que pleiteavam a despenalização, a projetos que visavam a caracterização da prática em crime hediondo. Portanto, com acirrados conflitos de posicionamentos entre eles, não houve alteração no Código Penal Brasileiro (Morais, et al., 2018).

Segundo a OMS, estima-se que ocorrem em torno de 80.000 mortes maternas relacionadas ao aborto inseguro por ano no mundo. No Brasil, o abortamento aparece como uma, das quatro causas mais frequentes de morte materna, sendo, em alguns estados, a causa primeira. Estatísticas apontam que 97\% dos abortos inseguros ocorreram nos países menos desenvolvidos, indicando pouco progresso na luta contra esta fonte evitável de morbidade e mortalidade maternas (Strefling, et al., 2015).

Acontecem 80.000 mortes maternas relacionadas ao abortamento por ano, no mundo. Como resultado desse cenário, no Brasil, a curetagem é o segundo procedimento obstétrico mais realizado nas unidades de internação da rede pública de serviços de saúde, sendo superado apenas pelos partos normais, sendo que o número de hospitalizações decorrentes de complicações pós-abortamento correspondem a 10\% do total de internações durante o ciclo gestacional, e 10\% de todas as mortes durante a gestação, está associada ao aborto e suas complicações (Batista, et al.,2017).

O aborto vem sendo consequência de vários fatores, entre eles: baixo nível de escolaridade, gravidez não planejada, planejamento familiar não eficaz, falta de parceiro fixo, uso indiscriminado de medicamentos, morbidades maternas e/ou fetais, precárias condições de atenção à mulher nos serviços de saúde, entre outros. Estudo sobre a tendência espaço temporal do aborto inseguro no Brasil entre 1996 e 2012, mostrou que embora tenha ocorrido redução progressiva desta prática em nível nacional, o aborto ainda configura-se grave e negligenciado no país (Maranhão, et al.,2016).

Pesquisas demostram que acontecem cerca de 50 milhões de abortos no mundo, anualmente. No Brasil, o aborto espontâneo ocorre em $8 \%$ a 10\% de todas as gestações confirmadas, chegando a 15\% entre abortos espontâneos e provocados (Ribeiro, et al., 2017). Estudos apontam que há uma maior prevalência de abortamento nos extremos da vida reprodutiva, especialmente após os 40 anos, e que $10 \%$ de todas as mortes durante a gestação estão associadas ao aborto e suas complicações (Cabral, 2017). 
Uma fonte de dados sobre a ocorrência de aborto no país são as internações hospitalares, disponíveis no Sistema de Informações Hospitalares (SIH). O Sistema registra as internações hospitalares no sistema público brasileiro, coletando-as com a finalidade administrativa de contabilizar procedimentos realizados em internações e controlar os custos para repasse às unidades de saúde executoras pelas secretarias municipais de saúde (Cardoso \& Vieira \& Saraceni, 2020).

Diante desta problemática torna-se relevante compreender as evidências que caracterizam o perfil socioeconômico e demográfico das mulheres que passaram pelo processo de aborto. Para tal fim deve-se analisar a situação de abortamentos ocorridos na macrorregião de Caxias no leste do Maranhão, caracterizar o perfil socioeconômico e demográfico dos dados e descrever os aspectos clínicos dos abortamentos.

Este estudo permitirá que gestores, administradores e profissionais da saúde, possam conhecer os fatores socioeconômicos e clínicos dos abortos ocorridos na macrorregião em estudo, assim como, o perfil das mulheres que passam por tal situação. A pesquisa contribuirá com conhecimento científico, promoção da "autonomia" da mulher, melhoria na qualidade dos programas de atenção à saúde sexual e reprodutiva, educação em saúde, planejamento familiar, pré-natal, e consequentemente, a prevenção da reincidência de gestações não planejadas e a redução da demanda e dos custos dos serviços de saúde destinados ao tratamento do processo abortivo.

A Pesquisa Nacional de Aborto demonstrou que ao término da vida reprodutiva, cerca de uma a cada cinco mulheres já haviam abortado, sendo que mais da metade destes abortos (59\%), ocorreram quando as participantes eram adolescentes ou jovens na faixa etária de 12 a 24 anos, necessitando de internação hospitalar em 50\% dos casos (Monteiro, et al., 2015). Segundo Montenegro \& Rezende (2017) a frequência de abortamentos precoces clinicamente reconhecidos em mulheres com idade de 20 a 30 anos é de 9 a 17\%, aumentando rapidamente para $20 \%$ na idade de 35 anos, $40 \%$ com 40 anos e $80 \%$ com 45 anos.

Segundo dados do Ministério da Saúde, no ano de 2005, foram notificados 1.054.242 abortos induzidos no Serviço de Informações Hospitalares (SIH) do SUS. No Brasil, somente no ano 2008, houve 215.000 hospitalizações do SUS por complicações de abortos, destes apenas 3230 foram abortos legais. Embora se verifique decréscimo desses números na última década, a taxa atual de 3,7 abortamentos por 100 mulheres, em idade reprodutiva, ainda é, muitas vezes, superior aos valores observados nos países da Europa Ocidental, onde o abortamento é legal, seguro e acessível (Silva, et al., 2014).

\section{Metodologia}

Estudo descritivo de série temporal, de base populacional, que descreveu o cenário do aborto na macrorregião Leste do Maranhão, utilizando dados públicos disponíveis para consulta e para download nos diversos sistemas de informação em saúde do Brasil. Para as informações de óbitos foram utilizados os dados da base do Tabnet DATASUS entre 2010 e 2018 . Os dados foram obtidos por meio de download da página de Internet do Departamento de Informática do SUS (DATASUS, 2020).

Os critérios de inclusão para a pesquisa foram: óbitos fetais com duração da gestação inferior a 22 semanas, compreendendo o estado do Maranhão na macrorregião em estudo selecionando os óbitos acontecidos nos municípios de Caxias, Codó, Coelho Neto, Coroatá, Lago da Pedra Presidente Dutra, Timon e outros municípios.

Para a identificação e análise dos óbitos por aborto foi considerada a causa básica do óbito, conforme estabelecido na Classificação Internacional de Doenças, 10ª revisão (CID-10, 2020). Foram usadas as seguintes categorias da CID-10: P02 (Feto ou recem nascido afetados por complicações da placenta, cordão umbilical e membranas), P20 (hipóxia intrauterina) e P95 (morte fetal de causa não especificada).

Não houve submissão ao Comitê de Ética em Pesquisa por se tratar de pesquisa com dados públicos do Ministério da Saúde. Os resultados foram expressos de forma agregada, sem possibilidade de identificação, de acordo com a Resolução $n^{o}$ 466/2012 do Conselho Nacional de Saúde (CNS, 2012). 


\section{Resultados}

Os resultados obtidos pelo estudo estão representados pelos Quadros 1, 2, 3, 4 e 5. O Quadro 1 demonstra o número de óbitos por residência na macrorregião leste segundo o ano do óbito, também observamos a porcentagem desse número de óbitos em cada ano sabendo em qual deles a porcentagem dos óbitos foi maior. O Quadro 2, expressa o número de óbitos fetais de acordo com a Lista de Mortalidade da Classificação Internacional de Doenças. O Quadro 3 exprime o perfil epidemiológico dos óbitos fetais. O Quadro 4 aponta o número de óbitos de acordo com os municípios da macrorregião em estudo e o Quadro 5 indica o número de óbitos segundo as categorias da Classificação Internacional de Doenças.

Quadro 1. Óbitos por residência na Macrorregião de Saúde segundo ano do óbito e com duração da gestação com menos de 22 semanas no período de 2010 a 2018.

\begin{tabular}{|c|c|c|}
\hline Ano & Óbitos Registrados & $\%$ \\
\hline 2010 & 09 & 3,89 \\
\hline 2011 & 22 & 9,52 \\
\hline 2012 & 23 & 9,95 \\
\hline 2013 & 27 & 11,6 \\
\hline 2014 & 45 & 19,48 \\
\hline 2015 & 28 & 12,12 \\
\hline 2016 & 23 & 9,95 \\
\hline 2017 & 27 & 11,6 \\
\hline 2018 & 27 & 11,6 \\
\hline Total & 231 & 100 \\
\hline
\end{tabular}

2111 Macrorregião Leste

Fonte: DATASUS (2020).

Segundo o ano do óbito na Macrorregião Leste, observou-se uma maior prevalência dos óbitos no ano de 2014 com o registro de 45 óbitos e uma porcentagem de $(19,48 \%)$ do total de óbitos 231, e uma menor prevalência no ano de 2010 com o registro de 09 óbitos e a porcentagem de (3,89\%) do total de óbitos. Houve um crescimento do número de óbitos nos anos de 2010 a 2014 e no período de 2015 a 2018 o número de óbitos ficou entre 23, 27 e 28.

Quadro 2. Óbitos por residência por Macrorregião de Saúde segundo a Lista de Mortalidade da Classificação Internacional de Doenças (CID-10) com duração da gestação com menos de 22 semanas no período de 2010 a 2018.

\begin{tabular}{|c|c|c|}
\hline \multicolumn{1}{|c|}{ List Mort CID-10 } & Óbitos & Registrados \\
\cline { 2 - 3 } meto ou Recém Nascido Afetados por fatores & 92 & 39,82 \\
\hline Transtornos relacionados à duração da gravidez & 05 & 2,16 \\
\hline Hipóxia Intrauterina e Asfixia ao nascer & 69 & 29,87 \\
\hline Transtornos hemo e hematológicos do feto & 01 & 0,43 \\
\hline Restante das afecções perinatais & 60 \\
\hline Malformações congênitas do sistema nervoso & 02 \\
\hline Malformações congênitas do sistema & 01 \\
\hline cardiovascular & 0,97 \\
\hline Outras malformações congênitas & 0,86 \\
\hline Total & 231 \\
\hline
\end{tabular}

Fonte: DATASUS (2020). 
Conforme a Lista de Mortalidade da Classificação Internacional de Doenças houve um maior número de óbitos fetais afetados por fatores maternos com o número de óbitos registrados de 92 e uma porcentagem de $(38,82 \%)$ do total de óbitos (231) e menor número de óbitos relacionados a transtornos hemorrágicos e hematológicos, malformações congênitas do coração e outras malformações congênitas com o número de óbito registrado 01 representando $(0,43 \%)$ do número total de óbitos.

Quadro 3. Perfil epidemiológico dos óbitos fetais por local de residência segundo Macrorregião de saúde e duração gestação: Menos de 22 semanas no período: 2010-2018.

\begin{tabular}{|c|c|c|c|}
\hline \multicolumn{2}{|c|}{ Macrorregião Leste } & Quantidade & $\%$ \\
\hline \multirow{3}{*}{ Por tipo de Gravidez } & Unica & 221 & 95,67 \\
\hline & Dupla & 08 & 3,46 \\
\hline & Ignorados & 02 & 0,86 \\
\hline \multirow{3}{*}{ Sexo do feto } & Masculino & 130 & 56,27 \\
\hline & Feminino & 90 & 38,96 \\
\hline & Ignorado & 11 & 4,76 \\
\hline \multirow{4}{*}{ Local de ocorrência } & Hospital & 206 & 89,17 \\
\hline & Domicílio & 14 & 6,06 \\
\hline & Outros & 08 & 3,46 \\
\hline & Ignorado & 03 & 1,29 \\
\hline \multirow{6}{*}{ Escolaridade } & Nenhuma & 16 & 6,92 \\
\hline & 1 a 3 anos & 19 & 8,22 \\
\hline & 4 a 7 anos & 69 & 29,87 \\
\hline & 8 a 11 anos & 90 & 38,96 \\
\hline & 12 e mais & 08 & 3,46 \\
\hline & Ignorados & 29 & 12,55 \\
\hline \multirow{3}{*}{ Tipo de parto } & Vaginal & 192 & 83,11 \\
\hline & Cesário & 37 & 16,01 \\
\hline & Ignorado & 02 & 0,86 \\
\hline \multirow{10}{*}{ Idade da mãe } & 10 a 14 anos & 03 & 1,29 \\
\hline & 15 a 19 anos & 59 & 25,54 \\
\hline & 20 a 24 anos & 48 & 20,77 \\
\hline & 25 a 29 anos & 49 & 21,21 \\
\hline & 30 a 34 anos & 31 & 13,41 \\
\hline & 35 a 39 anos & 15 & 6,49 \\
\hline & 40 a 44 anos & 06 & 2,59 \\
\hline & 45 a 49 anos & 01 & 0,43 \\
\hline & 50 a 54 anos & 01 & 0,43 \\
\hline & Idade ignorada & 18 & 7,79 \\
\hline Total & & 231 & $100 \%$ \\
\hline
\end{tabular}

Fonte: DATASUS (2020).

Com relação ao tipo de gravidez houve uma maior prevalência em gestações do tipo única com um total de 221 óbitos representando $(21,12 \%)$ dos óbitos totais 231 , seguindo houve uma maior quantidade de fetos do sexo masculino com uma quantidade de 130 representando ( 56,27\%) dos óbitos totais, a maior prevalência do número de óbitos de acordo com o local de ocorrência foram registrados no hospital com uma quantidade de 206 óbitos representando (89,17\%) dos óbitos totais, de acordo com a escolaridade $(38,96 \%)$ dos óbitos fetais estão relacionados às mães com escolaridade de 8 a 11 anos, houve também maior número dos casos de óbitos fetais em mães com idade de 15 a 19 anos, como mostra o Quadro 3. 
Quadro 4. Óbitos por residência por macrorregião de saúde segundo município com duração da gestação: Menos de 22 semanas no período: 2010-2018.

\begin{tabular}{|c|c|c|}
\hline Município & Número de óbitos & \% \\
\hline Caxias & 12 & 5,19 \\
\hline Codó & 19 & 8,22 \\
\hline Coelho Neto & 10 & 4,32 \\
\hline Coroatá & 11 & 4,76 \\
\hline Lago da Pedra & 10 & 4,32 \\
\hline Presidente Dutra & 16 & 6,92 \\
\hline Timon & 46 & 19,91 \\
\hline Outros municípios & 107 & 46,32 \\
\hline Total & 231 & 100 \\
\hline
\end{tabular}

Fonte: DATASUS (2020).

No tocante do número de óbitos por município houve um maior número de óbitos em Timon representando (19,91\%) dos óbitos totais da Macrorregião Leste, precedido respectivamente de Codó com (8,22\%) dos óbitos, Presidente Dutra representando (6,92\%), Caxias correspondendo a (5,19\%), Coroatá sendo (4,76\%) e demais municípios totalizando (46,32\%) dos óbitos.

Quadro 5. Óbitos por Residência na Macrorregião de Saúde segundo Categoria CID-10 com menos de 22 semanas Período: 2010-2018.

\begin{tabular}{|c|c|c|}
\hline Categoria CID-10 & Número de óbitos & \% \\
\hline $\begin{array}{c}\text { P 02 Feto ou recém-nascido afetado por complicações da } \\
\text { placenta, cordão umbilical ou membranas. }\end{array}$ & 67 & 29 \\
\hline P20 Hipóxia intrauterina. & 69 & 29,87 \\
\hline P95 Morte fetal de causa não especificada. & 55 & 23,80 \\
\hline Outras causas. & 40 & 17,31 \\
\hline
\end{tabular}

2111 Macrorregião Leste

Fonte: DATASUS (2020).

Consonante a categoria CID-10 há um maior número de óbitos fetais por hipóxia intrauterina totalizando 69 óbitos e equivalendo a $(29,87 \%)$ dos óbitos totais da Macrorregião Leste, seguindo houve 67 óbitos por feto ou recém-nascido afetados por complicações da placenta, do cordão umbilical e das membranas mostrando (29\%) dos óbitos totais, ainda podemos observar um alto número de morte fetal de causa não especificada, sendo 55 equivalendo a $(23,80 \%)$ dos óbitos totais da Macrorregião, destarte torna-se imprescindível a avaliação criteriosa dos óbitos.

\section{Discussões}

\subsection{O Perfil Epidemiológico das Mulheres Com Perda Gestacional}

A perda gestacional é a complicação mais comum da gestação, cerca de $20 \%$ das gravidezes clinicamente diagnosticadas evoluem para interrupção espontânea e não está associada somente a morbidade física ou alta mortalidade, mas, com repercussões sociais e psicológicas importantes à família. Há evidências de que o primeiro trimestre da gestação é o período onde há maiores perdas gestacionais, principalmente com IG entre a $8^{\mathrm{a}}$ e $12^{\mathrm{a}}$ semanas, sendo que $50 \%$ delas são decorrentes de anormalidades cromossômicas, com alterações numéricas ou estruturais, outras causas frequentes são a inviabilidade do desenvolvimento do feto e as alterações de implantação da placenta. 
Diversas intercorrências e alterações hormonais podem determinar a viabilidade ou inviabilidade do desenvolvimento do feto. Algumas intercorrências são determinadas através do acompanhamento do pré-natal, desde que este seja realizado de forma integral e a responsabilidade seja compartilhada entre profissionais de saúde e gestante no intuito de minimizar e prevenir intercorrências. Diversas intercorrências podem ser vistas na gestação e devem ser avaliadas de forma correta e realizado o tratamento o mais precoce possível, entre elas estão o acretismo e sua relação com a placenta prévia, a má suplementação da gestante nos três primeiros meses de gestação e consequentemente o rastreio de heranças genéticas e doenças hereditárias.

Os resultados obtidos através das análises dos quadros demonstram que vários fatores analisados na tentativa de determinar quais são os principais fatores inerentes ao aborto na macrorregião em estudo e os principais fatores analisados foram: a idade e escolaridade da mãe, o local de ocorrência do abordo, seja ele na residência, UBS, hospital ou outros; o tipo de gravidez, causas dos óbitos fetais de acordo com a lista de mortalidade da classificação internacional de doenças, ano do óbito e município de acordo com a macrorregião leste do Maranhão, com esses dados foi traçado um perfil das mulheres que passaram pelo processo de aborto.

O estudo mostra que houve maior número de óbitos fetais em mulheres entre 15 a 29 anos representando 67,52\% das mortes fetais, concordando com Cardoso et al. (2020) quando afirmam que a faixa etária de 20-29 anos foi a mais frequente entre as mulheres internadas com diagnóstico de aborto no Brasil por todo o período avaliado, e discordando do estudo de Soares e Cançado (2018) ao afirmarem que em relação à idade materna, 62,5\% das mulheres que tiveram perda gestacional com mais de 30 anos e deste total $60 \%$ são acima de 35 .

Outro fator interessante deste estudo são as principais causas analisadas nos últimos anos, com destaque nas causas maternas seja por práticas abortivas clandestinas ou por fatores biopsicossociais com 39,82\% dos casos, seguido por suprimento deficiente de oxigênio ou por asfixia no parto somando assim 29,87 dos casos e por último as afecções perinatais que são as intercorrências, inflamações e infecções no período perinatal com 25,97\% dos casos.

O estudo mostra que a escolaridade não é um indicador de reflexão acerca da mortalidade fetal, pois é evidenciado que mulheres com 8 a 11 anos de escolaridade estão com a maior quantidade do número de abortos nessa macrorregião de saúde sendo superior a quantidade de abortos das mulheres que não possuem nenhum grau de instrução, concordando com Soares e Cançado (2018) quando afirmam que à maioria das mulheres, que estiveram em processo abortivo, terminaram o $2^{\circ}$ grau de estudo.

Torloni et al. (2001) mencionaram em seus estudos que as causas diversas do aborto relacionam-se também a paridade elevada figura para alguns como possível fator predisponente ao acretismo, hipóxia e outros fatores inerentes as intercorrências gestacionais. Fatores estes que corroboram com o que diversos autores têm apontado que os sucessivos partos e dequitações talvez venham a comprometer o mecanismo regenerativo do endométrio, ou simplesmente aumentem a probabilidade de exposição a fatores predisponentes ao aborto.

Para Silva, et al. (2017) a gravidez é um período em que alterações fisiológicas, psíquicas, hormonais e sociais estão presentes, aumentando o risco de sofrimento emocional e morbidade psiquiátrica nesta fase da vida da mulher, corroborando com esta análise (Gonçalves, et al., 2014) investigou a prevalência de anormalidades cromossômicas em casais com dois ou mais abortos espontâneos recorrentes no primeiro trimestre de causa desconhecida, para determinar as possíveis e determinou que os processos de translocações ou arranjos genéticos possuem grandes impactos nas heranças genéticas e consequentemente, nos abortos por causas aparentemente desconhecidas.

Concordando com os autores citados anteriormente, Domingos e Mereghi (2010), afirma em seu estudo que o aborto é um tema polêmico e um sério problema de saúde pública mundial, responsável pela manutenção das altas taxas de mortalidade materna em muitos países em desenvolvimento. Para estes autores as complicações físicas do aborto podem estar presentes em 
decorrência do próprio processo de abortamento ou pelos procedimentos realizados para tratamentos. Essas complicações podem ser classificadas em grandes hemorragias, perfurações uterinas decorrentes de sondas ou cânulas, ulcerações do colo ou vagina por uso de comprimidos, infecções, esterilidade secundária a salpingite, salpingite crônica, algias pélvicas, transtornos menstruais e complicações obstétricas, tais como inserção anormal da placenta, abortamentos habituais, parto prematuro e entre outros. Todavia a realidade brasileira e mundial ainda é algo a se buscar melhorar no sentido de integrar e levar um olhar de equidade à gestante em processo abortivo e aos profissionais que as assiste.

Para Domingos e Mereghi (2010) e (Gonçalves, et al., 2014) a qualidade da atenção em saúde ofertada a mulheres que enfrentam esse processo é fundamental e implica a necessidade de um espaço integrado e sinérgico de todos os níveis gestores para a oferta de serviços que assegurem o acolhimento, informação, aconselhamento, com a responsabilidade e competência profissional, o uso de tecnologia apropriada disponível e relacionamento pessoal pontuado no respeito à dignidade e aos direitos sexuais e reprodutivo da mulher e nas liberdades conferidas na constituição e políticas de atenção a mulher e com frequência a necessidade de uma equipe que compreenda suas necessidades, não somente físicas, mas também sociais e psicológicas.

\section{2 Óbitos Registrados na Base de Dados}

De acordo com DATASUS (2020), houve um crescimento do número de abortos nos anos de 2010 a 2014 e posteriormente uma diminuição dessa quantidade nos períodos de 2014 a 2018, esses dados relativos aos casos notificados por internações hospitalares. O presente estudo revela também a grande quantidade de dados ignorados apontando a grande necessidade do adequado preenchimento dos dados no SIH dado que sem esse adequado preenchimento, os dados ficam insuficientes. Além disso, as bases de dados não concedem uma estimativa do número real de abortos visto que há uma grande demanda de abortos clandestinos que não são notificados, mas apesar da subnotificação, os dados disponíveis permitem traçar um perfil das mulheres que passaram pelo processo de aborto.

O estudo evidencia a alta necessidade de investimentos em estudos e pesquisas de campo nessa temática para uma melhor identificação dos casos não notificados concordando com Cardoso et al. (2020) quando diz que o número de internações por aborto identificados é bastante inferior às estimativas do número de abortos ocorridos no Brasil e que há uma limitação no estudo de não conseguir captar dados da ocorrência de abortos sem necessidade de internação.

Em suma, evidencia-se neste estudo que além dos fatores citados inerentes e intrínsecos ao aborto e intercorrências relacionadas, há também as práticas clandestinas de aborto e a mulher ao realizar este procedimento, esta guarda sua dor em silêncio ou, no máximo, compartilha com pessoas de sua intimidade; em geral, ela não encontra apoio nos serviços de saúde. Conceder oportunidade para que a mulher fale sobre suas emoções não só ajuda, mas fornece subsídios para o planejamento dos cuidados necessários e específicos equitário e holístico a mulher em transição e auxilio para que haja melhor qualidade de vida e planejamento familiar para minimizar intercorrências e práticas clandestinas, auxiliando assim a redução das altas taxas de morbimortalidade maternas e fetal.

\section{Considerações Finais}

As evidências da presente pesquisa sugerem que o abortamento na Macrorregião Leste do Maranhão é mais preponderante no município de Timon, em mulheres na juventude, de baixa ou média escolaridade, tendo ainda maior predomínio dos óbitos em fetos do sexo masculino, sendo gravidez do tipo única e tipo de parto vaginal, além da maioria dos registros acontecerem nos hospitais, dos anos de 2010 a 2018 houve um aumento do número de óbitos, ainda encontra-se maior primazia em óbitos relacionados a fatores maternos de acordo com a lista de mortalidade da CID-10 e de acordo com a 
categoria CID-10 por hipóxia intrauterina, observa-se um grande número de casos ignorados ressaltando a grande necessidade da notificação adequada e do preenchimento completo dos registros.

O aborto é um tema complexo em vários contextos, configurando-se um problema de saúde pública que necessita de uma maior relevância uma vez que atinge muitas mulheres inseridas nos mais diversos cenários socioeconômicos, ademais nota-se a insuficiência de informações no tocante às consequências deste procedimento e a alguns dados que necessitam de uma maior seriedade quanto à prestação de informações. Constata-se também a conveniência de uma atenção mais humanizada, e que não esteja centralizada apenas nas mulheres, mas, do mesmo modo na família que é o alicerce para auxiliar na atenuação desse acontecimento, portanto, é necessário implementar políticas públicas que informatizem as mulheres e à família quanto ao uso de preservativos e métodos anticonceptivos/contraceptivos para que se previna a gravidez não planejada e por conseguinte o aborto e seus reveses, e também políticas que visem estimular as famílias e as mulheres a buscar o atendimento do pré-natal na UBS para que se evite possíveis complicações que podem causar a morte tanto do feto como da mãe decorrentes da ausência de um serviço público e de fácil acesso como o pré-natal, para coactar a ocorrência de aborto.

Destarte, essa pesquisa dedica-se a evidenciar os fatos da realidade de muitas mulheres em situação de risco e vulnerabilidade que necessitam de uma maior alerta frente aos obstáculos a serem enfrentados diante da pobreza de informações, para que o governo juntamente aos órgãos com jurisdição sobre a saúde possam explorar o perfil socioeconômico das mulheres que passam pelo processo de aborto e elaborar políticas públicas voltadas para essa problemática, que estejam fixadas no objetivo de esclarecer todos os dados relativos às mulheres que passam por tal situação e as condições em que elas estavam antes, durante e após o acontecimento a fim de realizar a proteção, prevenção e promoção da saúde da mulher, proporcionando o progresso da saúde global, pois segundo a organização mundial da saúde "saúde é o completo bem estar físico mental e social", e esse impasse afeta não apenas o bem estar físico dessas mulheres, mas provoca uma série de problemas mentais e sociais às mesmas.

\section{Limitações e dificuldades encontradas}

Em decorrência da pandemia do novo coronavírus (Covid-19), não foi possível realizar a pesquisa de campo como esperado.

\section{Referências}

Azevedo, A. F. (2017). Direito ao aborto, gênero e a pesquisa jurídica em direitos fundamentais. Revista Latinoamericana, 1(26), 236-261. 10.1590/19846487.sess.2017.26.12.a

Batista, R. Q., \& Koch, D. F., \& Bispo, A. M. de S., \& Luciano, T. V., \& Velten, A. P. C. (2017). Descrição das internações por aborto no estado do Espírito Santo, Brasil. Revista Brasileira De Pesquisa Em Saúde/Brazilian Journal of Health Research, 18(2), 79-86. https://periodicos.ufes.br/rbps/article/view/15087 Bonassa, R.T., \& Rosa, M.I., \& Madeira, K., \& Simões, P.W. (2015). Caracterização de casos de internação por abortos complicados na macrorregião Sul Catarinense, Arq. Catarin. Med., 44(3), 88-100. https://www.researchgate.net/publication/331134842

Cabral, A. C. V. (2017). Guia de bolso de obstetrícia. (2a ed.),Atheneu.

Cardoso, B. P., \& Vieira, F. M. S. B., \& Saraceni V. (2020). Aborto no Brasil: O que dizem os dados oficiais. https://www.scielo.br/scielo.php?script=sci_arttext\&nrm=iso\&lng=pt\&tlng=pt\&pid=S0102-311X2020001305001. 10.1590/01002-311X00188718

Conselho Federal de Medicina. (2012). Resolução CFM $N^{o} \quad$ 1.989/2012. Brasília. https://portaldeboaspraticas.iff.fiocruz.br/wpcontent/uploads/2019/02/1989_20121.pdf

Conselho Nacional de Saúde. (2012) Resolução CNS No 466/2012. Brasília. https://conselho.saude.gov.br/ultimas_noticias/2 013/06_jun_14_publicada_resolucao.html

Datasus. (2020). Ministério da Saúde. https://datasus.saude.gov.br/transferencia-de-arquivos/

Desterro, R., \& Lima, S. M., \& França, L. G., \& Lima, R. C. D. S. M. (2015). Perfil clínico-epidemiológico de mulheres em situação de abortamento em uma maternidade pública de São Luís - MA. Rev. Investg. Bioméd., 7(1), 16-27. http://www.ceuma.br/portalderevistas/index.php/RIB/article/view/16/0. https://doi.org/10.24863/rib.v7i1.16 
Domingos, S. R. F., \& Mereghi, M. A. B. (2010). O aborto como causa de mortalidade materna: um pensar para o cuidado de enfermagem. Esc. Anna Nery, 14(p), 177-181, http://www.scielo.br/scielo.php?script=sci_arttext\&pid=S1414-81452010000100026\&lng=en\&nrm=iso. https://doi.org/10.1590/S141481452010000100026

Fleury, A. C. (2016). Direito à assistência e dignidade: uma discussão além da descriminalização do aborto. Revista Jurídica, 15(2), 19-33. http://periodicos.unievangelica.edu.br/index.php/revistajuridica/article/view/1953/1737

Gonçalves, R. O., \& Santos, W. V. B., \& Sarno, M., \& Cerqueira, B. A. V., \& Gonçalves, M. S., \& Costa, O. L. N. (2014). Anormalidades cromossômicas em casais com abortos recorrentes no primeiro trimestre. Rev. Bras. Ginecol. Obstet, 36(3), 113-117. http://www.scielo.br/scielo.php?script=sci_arttext\&pid=S0100-72032014000300113\&lng=en\&nrm=iso. https://doi.org/10.1590/S0100-72032014000300004

Henriques, J. P. (2016). A ineficiente política da criminalização do aborto: um estudo comparativo entre Brasil e Uruguai. FIDES, 8(1), 232-244. http://www.revistafides.ufrn.br/index.php/br/article/view/272/279

Maranhão, T. A., \& Gomes, K. R. O., \& Barros, I. C. (2016). Fatores preditores do abortamento entre jovens com experiência obstétrica. Rev. Bras. Epidemiol, 19(3), 494-508. https://www.scielo.br/j/rbepid/a/7MHWZ4F4VBbQNZjWFPmLvWH/?lang=pt. https://doi.org/10.1590/1980-5497201600030003

Morais, E. R. C., \& Aléssio, R. L. S., \& Coelho, L. S., \& Pontes, J. L. C. (2018). Aborto e bioética no Jornal Folha de São Paulo. Psicol. Pesqui. 12(1), 1-10

Monteiro, M. F. G., \& Adesse, L., \& Drezett, J. (2015). Atualização das estimativas da magnitude do aborto induzido, taxas por mil mulheres e razões por 100 nascimentos vivos do aborto induzido por faixa etária e grandes regiões. Reprod. $e$ Clim., 30(1), 11-18. https://periodicos.ufjf.br/index.php/psicologiaempesquisa/article/view/23384. https://doi.org/10.24879/201800120010089

Montenegro, C. A. B., \& Rezende, L. (2017). Rezende obstetrícia. (13a ed.), Guanabara Koogan.

Munakampe, M. N., \& Fwemba, I., \& Zulu, J. M. (2021). Associação entre nível socioeconômico e fecundidade entre adolescentes de 15 a 19 anos: uma análise da Pesquisa Demográfica de Saúde da Zâmbia 2013/2014 (ZDHS). Reprod Health 18, 182. https://doi.org/10.1186/s12978-021-01230-8

Pereira, F. A. (2018). Estudo sobre a legalização do aborto: prós, contra e a quem compete decidir acerca da descriminalização. Revista Científica UMC, 3(1), 1-15. http://seer.umc.br/index.php/revistaumc/article/view/171

Ribeiro, C. L., \& Albuquerque, F. O., \& Souza, A. R. (2017). Internações por aborto espontâneo: um retrato de sua ocorrência em Fortaleza. Enferm. Foco, 8(1), 37-41. http://revista.cofen.gov.br/index.php/enfermagem/article/view/584. https://doi.org/10.21675/2357-707X.2017.v8.n1.584

Silva, M. M., \& Castro, P. M., \& Theisen, C., \& Freitas, H. M. B., \& Colomé, J. S. (2014). Problemática do aborto em Santa Maria, Rio Grande do Sul: dados epidemiológicos. Disciplinarum Scientia, 15(2), 249-259. https://periodicos.ufn.edu.br/index.php/disciplinarumS/article/view/1086/1030

Silva, T.M., \& Martins, A.M. (2015). A legalização do aborto no Brasil ao longo da História: avanços e desafios. Revista Venezolana de Estudios de Lamujer, 20(44), 197-214

Silva, M. M. J., \& Nogueira, D. A., \& Clapis, M. J., \& Leite, E. P. R. C. (2017). Ansiedade na gravidez: prevalência e fatores associados. Rev. esc. enferm. USP, São Paulo, 51, e03253. doi: 10.1590/s1980-220x2016048003253

Soares, A. M., \& Cançado, F. M. A. A. (2018). Perfil de mulheres com perda gestacional. Rev Med Minas Gerais; 28: e-1930. 10.5935/2238-3182.20180072

Strefling, I. S. S., \& Filho, W. D. L., \& Demori, C. C., \& Soares, M. C., \& Santos, C. P. (2015). Cuidado de enfermagem à mulher em situação de aborto: revisão integrativa. Rev. Enferm., 5(1), 169-177. 10.5902/2179769212533

Torloni, M. R., \& Moron, A. F., \& Camano, L. (2001). Placenta Prévia: Fatores de risco para o Acretismo. Rev. Bras. Ginecol. Obstet, 23(7), 417-422. http://www.scielo.br/scielo.php?script=sci_arttext\&pid=S0100-72032001000700002\&lng=en\&nrm=iso. https://doi.or/10.1590/S0100-72032001000700002 